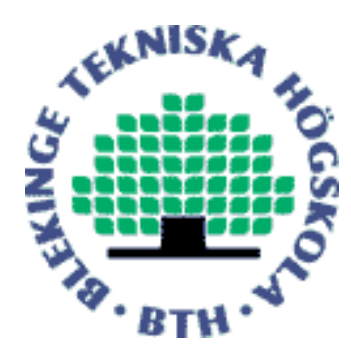

Electronic Research Archive of Blekinge Institute of Technology http://www.bth.se/fou/

This is an author produced version of a paper published in European Radar Conference, Munich 8-12 October 2007. This paper has been peer-reviewed but may not include the final publisher proof-corrections or journal pagination.

Citation for the published paper:

Mats I. Pettersson

Four-Dimensional Discretization for Detection of

Moving Objects in Wide Band SAR

European Radar Conference, Munich 8-12 October 2007

Access to the published version may

require subscription.

Published with permission from:

IEEE 


\title{
Four-Dimensional Discretization for Detection of Moving Objects in Wide Band SAR
}

\author{
M.I. Pettersson \\ Department of Signal Processing, Blekinge Institute of Technology \\ PO Box 520, SE-372 25 Ronneby, Sweden \\ mats.pettersson@bth.se
}

Abstract- This paper addresses four-dimensional Ground Moving Target Indication (GMTI) using a multi-channel Wide Band (WB) Synthetic Aperture Radar (SAR) system. With no acceleration attached to the target the motion of the target is related to two ground and two speed coordinates. However, four other dimensions are used in WB SAR GMTI processing during the detection phase: azimuth, range, bearing, and the relative speed between the object and the SAR platform. In the detection phase, blind hypotheses are used, and the discretization steps between the hypotheses are a trade-off between the number of hypotheses tested and detectability. As the integration angle increases, the step size in the image dimensions and in the relative speed has to be reduced. In this paper we determine the discretization step in all four dimensions for moving target detection, and relate it to radar system parameters. The discretization is derived from the moving target impulse response, assuming independency between the dimensions. In the paper the number of hypotheses per square meter is given for an airborne low frequency and a microwave GMTI WB SAR system.

\section{INTRODUCTION}

By combining SAR and array processing, clutter can by suppressed and moving targets detected. When long integration time is associated with the detection phase of moving targets, the target's range migration influences the radar system detection capabilities, and the target will be unfocused [1]. Different hypotheses of target motion are tested during the detection phase. With negligible acceleration during integration, the target movement is expressed by four dimensions, two speeds and two ground positions. Four other dimensions are measured by the radar system: range, azimuth, relative speed and bearing. The moving object will cause displacement in the SAR image, and the mismatch in relative speed will led to focusing loss and probably detection failures.

By determining the step size in all four dimensions it is possible to relate computation burden to detectability. A reduction in the target impulse response will cause a reduction in detectability.

This paper considers four dimensions of discretization: range, azimuth, relative speed and bearing. All dimensions are assumed to be independent. This approximation may not be fulfilled, however; the dependency can only reduce the number of hypotheses required in the test.

\section{MOVING OBJECT DETECTION}

Assuming a Cartesian coordinate system the range migration between the SAR system and the target is given by

$$
r_{t}(t)=\sqrt{\left(\xi_{p l}(t)-\xi_{t}(t)\right)^{2}+\left(\eta_{p l}(t)-\eta_{t}(t)\right)^{2}+\zeta_{p l}^{2}(t)}
$$

where $\left(\xi_{p l}(t), \eta_{p l}(t), \zeta_{p l}(t)\right)$ is the position of the platform and $\left(\xi_{t}(t), \eta_{t}(t), 0\right)$ is the position of the moving target at time $t$. With linear motion, the position of the platform is $\left(v_{p l} t, 0, h\right)$ and the moving target position $\left(v_{\xi} t+\xi_{0}, v_{\eta} t+\eta_{0}, 0\right)$. Where $\left(v_{p}, 0,0\right)$ is the platform speed, $\left(v_{\xi}, v_{\eta}, 0\right)$ is the target speed vector and $h$ is the flight height. These equations assumes no acceleration, and therefore the range distance can be expressed [2] as

$$
r_{t}(t)=\sqrt{\gamma_{t}^{2}\left(v_{p l} t-x_{t}\right)^{2}+y_{t}^{2}}
$$

where $x_{t}$ and $y_{t}$ are the moving target position in the processed SAR image and $\gamma_{t}$ is related to the relative speed between the SAR platform and the moving target. With a linear motion model, the multi-dimensional problem is simplified to a three-dimensional problem given by $x_{t}$, $y_{t}$ and $\gamma_{t}$. However, the true target position in four dimensions $\left(\xi_{0}, \eta_{0}, 0\right)$ and speed coordinates $\left(v_{\xi}, v_{\eta}, 0\right)$ cannot be established using the three dimensions $x_{t}, y_{t}$ and $\gamma_{t}$. The relations between the true target position and the measured parameters are given by [2]

$$
\begin{gathered}
x_{t}=\xi_{0}-\frac{v_{\eta}}{v_{p}-v_{\xi}} \eta_{0} \\
y_{t}=\sqrt{\eta_{0}^{2}\left(1+\left(\frac{v_{\eta}}{v_{p}-v_{\xi}}\right)^{2}\right)+h^{2}} \\
\gamma_{t}=\sqrt{\left(v_{p}-v_{\xi}\right)^{2}+v_{\eta}^{2}} / v_{p}
\end{gathered}
$$

When estimating $\left(x_{t}, y_{t}, \gamma_{t}\right)$ the target parameters $\left(\xi_{0}, \eta_{0}, v_{\xi}, v_{\eta}\right)$ will remain undecided, e.g. estimating the true relative speed gives a circle of possible speeds in $\left(v_{\xi}, v_{\eta}, 0\right)$ 
in (5). In (3) and (4), the unknown speed relation $v_{\eta} /\left(v_{p}-v_{\xi}\right)$ will cause uncertainties in the ground coordinates. To establish true position, a fourth dimension in space time has to be measured, normally by an antenna array with elements in the SAR system moving direction. The fourth dimension also adds clutter suppression capabilities. The fourth dimensional test is given by [2]

$$
\begin{aligned}
& \left|\int_{-\infty}^{\infty} \int_{k_{\min }}^{k_{\max }} e^{j k k_{r}\left(t_{s}\right)} \mathbf{A}^{H}\left(\varphi_{h}^{\prime}\left(t_{s}\right), k, t_{s}\right) \mathbf{C}^{-1}\left(k, t_{s}\right) \tilde{\mathbf{Y}}\left(k, t_{s}\right) d k d t_{s}\right|^{2}= \\
& \quad=\left\{\begin{array}{l}
\geq \lambda \text { decision for } \mathbf{H}_{1} \\
<\lambda \text { decision for } \mathbf{H}_{0}
\end{array}\right.
\end{aligned}
$$

where $\tilde{\mathbf{Y}}\left(k, t_{s}\right)$ is measurement vector, $\mathbf{C}\left(k, t_{s}\right)$ is the covariance matrix, $k$ is the wave number, $\mathbf{A}\left(\varphi_{h}^{\prime}\left(t_{s}\right), k, t_{s}\right)$ is the steering vector for the moving target given by

$$
\mathbf{A}\left(\varphi_{h}^{\prime}\left(t_{s}\right), k, t_{s}\right)=\left[\begin{array}{c}
1 \\
e^{-j k d \delta_{h}\left(t_{s}\right)} \\
\vdots \\
e^{-j k(N-1) d \delta_{h}\left(t_{s}\right)}
\end{array}\right]
$$

where

$$
\delta_{h}\left(t_{s}\right)=\cos \varphi_{h}\left(t_{s}\right)-\cos \varphi_{h}^{\prime}\left(t_{s}\right)
$$

and $d$ the distance between the effective antenna phase centers in the array, $\varphi\left(t_{s}\right)$ is the Doppler angle, $\varphi_{t}^{\prime}\left(t_{s}\right)$ is the real angle between the flight track and the moving target and $N$ is the number of antenna channels.

\section{HYPOTHESIS OF RANGE, AZIMUTH, RELATIVE SPEED AND BEARING}

For detection, different hypotheses on range migration and bearing are tested in (6). The hypothesis of range migration is given by

$$
r_{h}\left(t, \gamma_{p}, x_{h}, y_{h}\right)=\sqrt{\gamma_{p}^{2}\left(v_{p l} t-x_{h}\right)^{2}+y_{h}^{2}}
$$

where $\gamma_{p}$ is the hypothesis of relative speed and $x_{h}$ and $y_{h}$ are the image position under test. These three dimensions are related to the geometry between the platform and the target. The fourth dimension is given by the angle separation of target and its SAR appearance $\delta_{h}\left(t_{s}\right)$ in (8) measured by the array antenna. To secure detection in equation (6), the shape of the impulse response in four dimensions must be determined. In this paper, the discretization is derived from loss in the discretization compared to the peak value in the impulse response. The moving target peak value in the SAR image impulse response will be maximized when $\gamma_{p}=\gamma_{t}, x_{h}=x_{t}, y_{h}=y_{t}$ and $\delta_{h}=\delta_{t}$.

\section{DISCRETIZATION IN IMAGE COORDINATES $x_{h}$ AND $y_{h}$.}

The resolution in the SAR image is given by the radar bandwidth and the integration angle. The resolution in a WB SAR system can be found in [3]. To simplify the calculations we assume the integration angle to be symmetric around broadsight. The resolution in range is given by the system bandwidth; a good approximation for the amplitude is [4]

$$
h\left(\gamma_{t}, x_{t}, y_{h}, \varphi_{t}^{\prime}\right) \approx \operatorname{sinc}\left(\frac{2 \pi B\left(y_{h}-y_{t}\right)}{c}\right)
$$

with $\operatorname{sinc}(x)=\sin (x) / x$ and in azimuth

$$
h\left(\gamma_{t}, x_{t}, y_{h}, \varphi_{t}^{\prime}\right) \approx \operatorname{sinc}\left(\frac{2 \pi \Delta \vartheta \gamma_{h}\left(x_{h}-x_{t}\right)}{\lambda_{c}}\right)
$$

This approximation assumes a flat frequency dependency in the wave domain. However, the clutter filtering in (6) will influence the spectra; this has not been taken into consideration in the present research. Nevertheless, the derived step size using flat spectra will yield a smaller step size than the actual filtered one; there will thus be lower loss and more secure detection.

\section{DisCRETIZATION OF CHANNEL SEPARATION $\delta_{h}\left(t_{s}\right)$}

The discretization of the channel separation is limited by antenna channel spatial separation, number of channels and operating frequency. The impulse response at $k$ and $t_{s}$ is given by

$$
\mathbf{A}^{H}\left(\varphi_{h}^{\prime}\left(t_{s}\right), k, t_{s}\right) \mathbf{C}^{-1}\left(k, t_{s}\right) \tilde{\mathbf{Y}}\left(k, t_{s}\right)
$$

In this paper we approximate the discretization in all four dimensions. The strong clutter in the covariance matrix will broaden the impulse response in the angular domain. However, to be confident of detection we assume that the array function is given by [5]

$$
\mathrm{AF}\left(k, \delta_{h}(t)\right)=\frac{\sin \left(\frac{N}{2} k d \delta_{h}(t)\right)}{N \sin \left(\frac{1}{2} k d \delta_{h}(t)\right)}
$$


The width is dependent on the wave number in (13). Considering a WB SAR the width changes over the integration interval of $k$ in (6). Because the smallest width is associated with the largest $k$, we select $k=k_{\max }$ in equation (6) in order to ensure secure detection, and we rewrite (6) as

$$
\left|\int_{-\infty}^{\infty} g\left(t, r_{h}\left(t_{s}, \gamma_{p}, x_{h}, y_{h}\right)\right) \operatorname{AF}\left(k_{\max }, \delta_{h}\left(t_{s}\right)\right) d t_{s}\right|^{2}
$$

\section{DISCRETIZATION IN RELATIVE SPEED}

If $\gamma_{h} \neq \gamma_{t}$, the unfocused moving target will cause a peak value decrease in the target's impulse response as compared to $\gamma_{p}=\gamma_{t}$. To estimate the decrease based on relative speed we use equation (14). In reality, there is a limitation for the linear motion model; the maximum integration time has to be selected according to priori information. Assuming integration time $t_{i}$, the test in (14) is given approximately by

$$
\left|\int_{t_{0}-t_{i} / 2}^{t_{0}+t_{i} / 2} g\left(t, r_{h}\left(t_{s}, \gamma_{p}, x_{h}, y_{h}\right)\right) d t_{s} \operatorname{AF}\left(k_{\max }, \delta_{h}\left(t_{o}\right)\right)\right|^{2}
$$

The angular domain dependency is then separated from the other dimensions, and the derived step size becomes smaller in the approximate solution as compared to the exact solution. Identifying the global backprojection integral in (15) we get

$$
h\left(\gamma_{p}, x_{h}, y_{h}\right)=\int_{t_{0}-t_{i} / 2}^{t_{0}+t_{t} / 2} g\left(t, r_{h}\left(t, \gamma_{p}, x_{h}, y_{h}\right)\right) d t
$$

The ratio between the hypotheses being tested and the focused target is given by

$$
h_{\text {ratio }}\left(t_{0}, \gamma_{p}\right)=\frac{\int_{t_{0}-t_{i} / 2}^{t_{0}+t_{t} / 2} g\left(t, r_{h}\left(t, t_{0}, \gamma_{h}\right)\right) d t}{\int_{t_{0}-t_{i} / 2}^{t_{t} / 2} g\left(t, r_{t}(t)\right) d t}
$$

for a transmitted chirp pulse, (17) can be approximated to [6]

$$
h_{\text {ratio }}\left(t_{0}, \gamma_{p}\right) \approx{\frac{1}{t_{i}}}_{t_{0} t_{i} / 2}^{t_{0}+t_{i} / 2} e^{-j 4 \pi f_{c} \frac{\Delta r\left(t, t_{0}, \gamma_{p}\right)}{c}} \operatorname{sinc}\left(\frac{2 \pi B \Delta r\left(t, t_{0}, \gamma_{p}\right)}{c}\right) d t
$$

where $\Delta r\left(t, t_{0}, \gamma_{p}\right)=r_{h}\left(t, t_{0}, \gamma_{p}\right)-r_{t}(t)$.
The ratio $h_{\text {ratio }}\left(t_{0}, \gamma_{p}\right)$ is reduced with integration time $t_{i}$, and even for a narrow-band SAR system the ratio decreases drastically as $\Delta r\left(t, t_{0}, \gamma_{p}\right)$ gets in length of the radar system operating wave length $\lambda_{c}=c / f_{c}$. According to [6], (17) can be rewritten as

$$
h_{\text {ratio }}(Q, q) \approx \frac{1}{2 Q} \int_{-Q}^{Q} e^{-j p^{2}} \operatorname{sinc}\left(\frac{q p^{2}}{2}\right) d p
$$

where $q=B / f_{c}$ is the relative bandwidth. Knowing $q$ and the maximum acceptable loss (19) can be calculated. The optimum quantization step is found to be

$$
\Delta \gamma_{p}=\sqrt{\gamma_{t}^{2}+\frac{2 Q^{2}}{\pi \chi^{2}}}-\gamma_{t}
$$

where $\chi$ is the radar-dependent parameter $\chi=L / \sqrt{\lambda_{c} r\left(t_{0}\right)}$. The integral in (19) is a good approximation of (17) when the used aperture is much smaller then the target minimum distance $L<<y_{t}$. However, [6] demonstrates that it is also a good approximation for large apertures.

\section{RESULTS}

The detectability is limited by the peak value in the test (6). However, searching in the four dimensional space only discrete points can be tested and there will be an energy loss between the peak value and the closest test point. Larger discretization step is associated with larger loss and in this paper we determine the discretization step relation to the energy loss. If the maximum loss is $\varepsilon_{\text {total }}$, and if we assume four independent dimensions, the loss in each dimension is given by $\varepsilon_{\text {total }} / 4$.

The impulse response in the four dimensions is given by

$$
\begin{gathered}
\left|\operatorname{sinc}\left(\frac{2 \pi B\left(y_{h}-y_{t}\right)}{c}\right)\right|^{2} \leq \frac{\varepsilon_{\text {total }}}{4} \\
\left|\operatorname{sinc}\left(\frac{2 \pi \Delta \vartheta \gamma_{p}\left(x_{h}-x_{t}\right)}{\lambda_{c}}\right)\right|^{2} \leq \frac{\varepsilon_{\text {total }}}{4} \\
\left|\operatorname{AF}\left(k_{\max }, \delta_{h}\left(t_{0}\right)\right)\right|^{2} \leq \frac{\varepsilon_{\text {total }}}{4} \\
\left|h_{\text {ratio }}(Q, q)\right|^{2} \leq \frac{\varepsilon_{\text {total }}}{4}
\end{gathered}
$$


With a total loss of $3 \mathrm{~dB}$, which is $0.75 \mathrm{~dB}$ in each dimension, the step size in the image space is given by $\Delta y \approx 1.4 c /(\pi B)$ and $\Delta x \approx 1.4 \lambda_{c} /\left(\pi \gamma_{p} \Delta \vartheta\right)$.

In this paper we consider two different radar systems with the same geometric properties carried by, for example, a medium altitude UAV (Unmanned Aerial Vehicle). The two systems are a microwave system operating at $15 \mathrm{GHz}$, and a low-frequency system operating at $500 \mathrm{MHz}$. For the microwave system, we assume an integration time of $1 \mathrm{~s}$ as compared to the integration time of $10 \mathrm{~s}$ in the lowfrequency system. Table I gives the parameters for each system. If, for example, the integration time is longer or the distance changes, the discretization step will also change.

TABLE I.

\begin{tabular}{|l|l|l|l|}
\hline \multicolumn{3}{|c|}{ Radar system parameters } & \\
\hline Radar parameters & & $\begin{array}{l}\text { Low } \\
\text { frequency }\end{array}$ & Microwave \\
\hline Minimum distance & & $5000 \mathrm{~m}$ & $5000 \mathrm{~m}$ \\
\hline Platform speed & $v_{p}$ & $100 \mathrm{~m} / \mathrm{s}$ & $100 \mathrm{~m} / \mathrm{s}$ \\
\hline Centre frequency & $f_{c}$ & $500 \mathrm{MHz}$ & $15 \mathrm{GHz}$ \\
\hline Relative bandwidth & $q$ & 1 & 0.1 \\
\hline Integration time & $t_{i}$ & $10 \mathrm{~s}$ & $1 \mathrm{~s}$ \\
\hline $\begin{array}{l}\text { Antenna effective } \\
\text { distance }\end{array}$ & $d$ & $1 \mathrm{~m}$ & $0.5 \mathrm{~m}$ \\
\hline Number of channels & $L$ & 3 & 3 \\
\hline $\begin{array}{l}\text { Maximum discretization } \\
\text { loss }\end{array}$ & $\varepsilon_{\text {total }}$ & $3 \mathrm{~dB}$ & $3 \mathrm{~dB}$ \\
\hline Loss in each dimension & & $0.75 \mathrm{~dB}$ & $0.75 \mathrm{~dB}$ \\
\hline
\end{tabular}

Table II gives the discretization step in all four dimensions, according to the system parameters given in Table I.

TABLE II.

\begin{tabular}{|l|l|l|l|l|}
\hline \multicolumn{5}{|c|}{ Disctretization } \\
\hline Radar system & $\Delta x$ & $\Delta y$ & $\Delta \delta_{h}$ & $\Delta \gamma_{h}$ \\
\hline Low frequency & $0.68 \mathrm{~m}$ & $0.14 \mathrm{~m}$ & 0.064 & 0.0025 \\
\hline $\begin{array}{l}\text { Microwave } \\
\text { system }\end{array}$ & $0.23 \mathrm{~m}$ & $0.045 \mathrm{~m}$ & 0.0016 & 0.0061 \\
\hline
\end{tabular}

Assume that we have an area of interest where the two radar systems are operating, and that target speed is less then $10 \mathrm{~m} / \mathrm{s}$. The channel separation is then limited by $\left|\delta_{h}^{\max }\right| \leq 0.1$ for the low frequency system and $\left|\delta_{h}^{\max }\right| \leq 0.05$ for the microwave system. The total number of discretization levels in the angular domain is 4 and 62, respectively. In the relative speed domain, the interval of relative speed is given by $0.9 \leq \gamma_{h} \leq 1.1$, and the number of discretization levels are 166 and 45, repectively. In this example, the total number of tests needed per square meter for the low-frequency system is 2700 hypotheses $/ \mathrm{m}^{2}$, and for the microwave system, 72000 hypotheses $/ \mathrm{m}^{2}$. If the area of interest is large, the total number of tests will be enormous. However, the high resolution given in Table II is probably not necessary in the detection phase, because moving targets are often 3 meters or larger. This fact, along with a reduction of the $3 \mathrm{~dB}$ resolution to $3 \mathrm{x} 3$ meters, makes it possible to radically reduce the number of tests. Using the loss $0.75 \mathrm{~dB}$ in every dimension the number of tests per square meter is reduced to more reasonable levels. For the low frequency system, the number of tests is 13 , and for the high frequency it is 7 per square meter. Because the number of tests associated with relative speed is much larger for the low frequency system, it is expected that detection when applied to this system will require greater computation power. This computational burden should be placed in relation to the low frequency system capabilities to detect targets in forested areas. However, in [7] a method is proposed to process moving targets in the relative speed domain that will reduce demands on computational power. However, this field requires greater research.

\section{CONCLUSION}

In this paper we derive the discretization step between hypotheses in four dimensions needed for moving target detection using a GMTI SAR system. The step is derived by considering the loss in object peak response and independency between the dimensions. The number of discretization tests needed per square meter is calculated for two different WB radar systems. By increasing resolution in the system, the number of tests increases drastically.

\section{ACKNOWLEDGEMENT}

The author would like to thank the KK Foundation for its generous funding which has made the present research project possible. Thanks also go to Saab Bofors Dynamics, Saab Microwave System and Saab Space.

\section{REFERENCES}

[1] R. K. Raney: "Synthetic Aperture Imaging Radar and Moving Targets”, IEEE Trans. Aerospace and Electronic Systems, Vol. AES-7, No. 3, pp. 499-505, 1971

[2] M.I. Pettersson, "Detection of moving targets in wideband SAR", IEEE Trans. Aerospace and Electronic systems, vol 40, no.3 , pp. 780-796, July 2004

[3] L. M. H., Ulander, and H., Hellsten, "A new formula for SAR spatial resolution”, AEU International Journal of Electronic Communication, vol. 50, no. 2, pp. 117-121, 1996

[4] C. Oliver, S., Quegan, Understanding Synthetic Aperture Radar Images, London: Artech House, 1998

[5] C.A. Balanis, Antenna Theory - Analyses and Design, 2nd ed., Hobocken USA: John Wiley \& Sons, INC.; 1997

[6] M.I. Pettersson, IET Radar, Sonar \& Navigation, accepted

[7] H. Hellsten.; L.M.H. Ulander; "Airborne array aperture UWB UHF radar-motivation and system considerations”, Aerospace and Electronic Systems Magazine”, IEEE, Vol. 15, Issue 5, pp 35-45, 2000 